\title{
Axisymmetric Wave Propagation Behavior in Fluid-Conveying Carbon Nanotubes Based on Nonlocal Fluid Dynamics and Nonlocal Strain Gradient Theory
}

\author{
Yang Yang ${ }^{1} \cdot$ Qihui Lin ${ }^{1} \cdot$ Rongxin Guo ${ }^{1}$
}

Received: 31 October 2019 / Revised: 20 December 2019 / Accepted: 23 December 2019 / Published online: 29 January 2020

(c) The Author(s) 2020

\begin{abstract}
Purpose Goal for the present research is investigating the axisymmetric wave propagation behaviors of fluid-filled carbon nanotubes (CNTs) with low slenderness ratios when the nanoscale effects contributed by CNT and fluid flow are considered together.

Method An elastic shell model for fluid-conveying CNTs is established based on theory of nonlocal elasticity and nonlocal fluid dynamics. The effects of stress non-locality and strain gradient at nanoscale are simulated by applying nonlocal stress and strain gradient theories to CNTs and nonlocal fluid dynamics to fluid flow inside the CNTs, respectively. The equilibrium equations of axisymmetric wave motion in fluid-conveying CNTs are derived. By solving the governing equations, the relationships between wave frequency and all small-scale parameters, as well as the effects caused by fluid flow on different wave modes, are analyzed.

Results The numerical simulation indicates that nonlocal stress effects damp first-mode waves but promote propagation of second-mode waves. The strain gradient effect promotes propagation of first-mode waves but has no influence on secondmode waves. The nonlocal fluid effect only causes damping of second-mode waves and has no influence on first-mode waves. Damping caused by nonlocal effects are most affect on waves with short wavelength, and the effect induced by strain gradient almost promotes the propagation of wave with all wavelengths.
\end{abstract}

Keywords Nonlocal fluid dynamics $\cdot$ Nonlocal strain gradient theory $\cdot$ Fluid-conveying carbon nanotubes $\cdot$ Axisymmetric wave propagation $\cdot$ Elastic shell model

\section{Introduction}

Since the development of application about micro/nano-electro-mechanical systems (M/NEMS) in chemical, medicine and mechanical engineering, fluid transmission at microand nanotubes or channels attracts lots of research interests. The key factor for the design and production of such fluid transmission structures is the fluid-structure interaction at the micro- or nanoscale, especially the dynamic behavior for fluid-conveying CNTs [1-3].

Yang Yang

yangyang0416@kust.edu.cn

1 Key Laboratory in Yunnan Province for Disaster Reduction of Civil Engineering, Kunming University of Science and Technology, Kunming 650500, China
Theoretical analysis and experimental research are two general approaches for studying the dynamic characteristics of fluid-conveying CNTs. However, dynamic experimentation at the nanoscale is quite difficult to execute and control [2]; many theoretical and numerical methods have been employed, including molecular dynamics simulations (MD) $[4,5]$, classical elastic constitutive models [6], and smallscale elastic models such as the coupled stress model, nonlocal elastic model and elastic strain gradient model [7-16]. MD is the most reasonable approach because it calculates the interaction between all of the atoms in the system, but the application of MD is limited to some complex structures, such as fluid-conveying systems. Classical elastic models could provide reasonable results without complicated calculations. However, some dynamic properties predicted by classical models are inaccurate since the size effects at nanoand microscales are ignored. Therefore, continuum models based on small-scale theory are the most appropriate tool for 
analysis of micro- or nanoeffects on the mechanical properties of nanostructures. One of this continuum models is the nonlocal elastic model proposed by Eringen [17].

Since nonlocal constitutive equation is simple for calculation, many nonlocal continuum models have been established to study the dynamic properties of CNTs [8-16]. However, the nonlocal constitutive equation can only predict the scale effects of solids, when fluid flow inside CNT must be modeled by other theories, such as the slip boundary theory [14-16]. Thus, some unsatisfactory results are obtained, because slip boundary condition is inappropriate for liquids. Furthermore, the nonlocal constitutive equation for solids is also inadequate in some cases. For example, analyses of high-frequency wave propagation based on nonlocal beam and shell models are not accurate [10].

To correct the deficiencies of the nonlocal model, Lim and his colleagues developed a new constitutive equation that coupled two kinds of scale effect caused by stress nonlocality and strain gradients in a single combined equation [18]. By applying this new stress/strain equation, different kinds of nano- and micro-scale effects caused by strain and stress can be analyzed together, and many numerical results related to the dynamic behavior of nanostructures have been published on the basis of this coupling nonlocal strain gradient model.

Li C and his colleague employed the nonlocal strain gradient elastic models to predict the dynamic behaviors of nanostructures, and found the stress nonlocality causes a softening effect when the strain gradient leads to a stiffening result [19-21]. Li L et al. applied this new model to study the vibration behavior for fluid-filled nanotubes, while more exact results were obtained than other numerical simulation methods [22-24]. Zeighampour et al. established beam and shell models based on Lim's theory [18] to investigate dynamic properties of double-walled fluid-conveying carbon nanotubes; while the slip boundary theory is employed to analyze the critical fluid velocity [25-30]. However, most of the numerical results obtained by these slip boundary models are not satisfactory, since the slip boundary condition is inappropriate for nano- or microscale liquid. To overcome this shortcomings, the authors simulated the nanoscale effects induced by fluid flow based on nonlocal fluid dynamics [17], while the dynamic nonlocal strain gradient beam models for fluid-conveying CNTs were developed [28, 29]. It was found the dynamic performances of fluid-conveying CNTs predicted by the new nonlocal fluid dynamic models agreed more closely with Monte Carlo simulation results than other continuum models, including slip boundary models [31, 32]. However, the beam models fail to analyze the CNTs with low slenderness ratios.

To predict dynamic properties of fluid-filled CNTs with low slenderness ratios, a nonlocal strain gradient shell model is developed to study the axisymmetric wave propagation characteristics. The equilibrium equation of axisymmetric wave motion in the fluid-conveying CNTs is obtained. The nanoscale effects leaded by fluid flow are simulated when using nonlocal fluid dynamics proposed by Eringen [17]. Thus the stress nonlocality and strain gradient induced by CNT and inner fluid are fully investigated.

\section{Dynamic Model for a Fluid-Filled Nanotube}

The fluid-conveying CNT is seen as a cylindrical shell in the Cartesian coordinate system, as indicated in Fig. 1, where fluid flow through the shell with uniform velocity $U$. The length and radius of CNT are $L_{0}$ and $R$, respectively.

According to Lim's theory [18], the elastic constitutive relation of a fluid-filled membrane shell are shown in Eqs. $(1-3)$ as follows [18]:

$\left[1-(\mathrm{ea})^{2} \nabla^{2}\right] \sigma_{x}=\lambda\left(1-l^{2} \nabla^{2}\right)\left(\varepsilon_{x}+\varepsilon_{\theta}\right)+2 \mu\left(1-l^{2} \nabla^{2}\right) \varepsilon_{x}$,

$\left[1-(e a)^{2} \nabla^{2}\right] \sigma_{\theta}=\lambda\left(1-l^{2} \nabla^{2}\right)\left(\varepsilon_{x}+\varepsilon_{\theta}\right)+2 \mu\left(1-l^{2} \nabla^{2}\right) \varepsilon_{\theta}$

$\tau_{x \theta}=2 \mu \varepsilon_{x \theta}$,

where $\sigma_{x}, \sigma_{\theta}$, and $\tau_{x \theta}$ are stress components along different directions; $\varepsilon_{x}, \varepsilon_{\theta}$, and $\varepsilon_{x \theta}$ are corresponding strain components; and $\lambda$ and $\mu$ denote elastic constants. The scale effects in Eqs. $(1,2)$ are calculated by the nonlocal parameter ea, and the strain gradient parameter $l$. Thus the classical constitutive equations are obtained when ea $=l=0$. The internal forces of the shell are obtained by integrating the stress tensor at the cross section of the shell as follows [33]:

$N_{x}, N_{\theta}, N_{x \theta}, N_{\theta x}=\int_{h / 2}^{-h / 2}\left(\sigma_{x}, \sigma_{\theta}, \tau_{x \theta}, \tau_{\theta x}\right) \mathrm{d} z$

where $h$ stands for the thickness of wall for CNT. Substituting Eqs. (1-3) into Eq. (4) yields

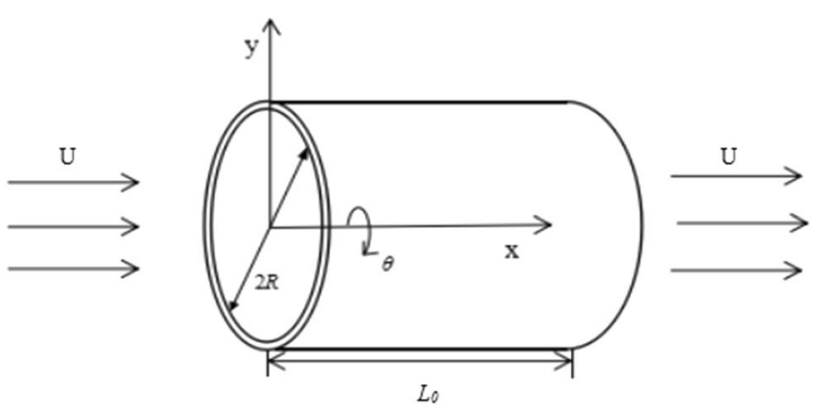

Fig. 1 Shell model of fluid-conveying CNT in the Cartesian coordinate system 
$\mu=E / 2(1+v), \lambda=E v /\left(1-v^{2}\right), \gamma=2 \varepsilon_{x \theta}$,

$N_{x}-(\text { ea })^{2} \frac{\partial^{2} N_{x}}{\partial x^{2}}=\frac{\mathrm{Eh}}{1-v^{2}}\left[\varepsilon_{x}-l^{2} \frac{\partial^{2} \varepsilon_{x}}{\partial x^{2}}+v\left(\varepsilon_{\theta}-l^{2} \frac{\partial^{2} \varepsilon_{\theta}}{\partial x^{2}}\right)\right]$,

$N_{\theta}-(\mathrm{ea})^{2} \frac{\partial^{2} N_{\theta}}{\partial x^{2}}=\frac{\mathrm{Eh}}{1-v^{2}}\left[\varepsilon_{\theta}-l^{2} \frac{\partial^{2} \varepsilon_{\theta}}{\partial x^{2}}+v\left(\varepsilon_{x}-l^{2} \frac{\partial^{2} \varepsilon_{x}}{\partial x^{2}}\right)\right]$,

$N_{x \theta}=N_{\theta x}=G h \gamma$,

where $\mu=E / 2(1+v), \lambda=E v /\left(1-v^{2}\right)$ and $\gamma=2 \varepsilon_{x \theta}$. The parameters $E, v$ and $\gamma$ stand for Young's modulus, Poisson's ratio and shear strain, respectively. Since the bending moments are negligible for a membrane cylindrical shell, the dynamic equilibrium equations for a fluid-conveying nanotube in the axial, circumferential and radial directions $(x, \theta$, and $r)$ are [34]

$\rho_{\mathrm{c}} h \frac{\partial^{2} u}{\partial t^{2}}-\frac{\partial N_{x}}{\partial x}-\frac{1}{R} \frac{\partial N_{\theta x}}{\partial \theta}=0$,

$\rho_{\mathrm{c}} h \frac{\partial^{2} v}{\partial t^{2}}-\frac{1}{R} \frac{\partial N_{\theta}}{\partial \theta}-\frac{\partial N_{x \theta}}{\partial x}=0$

$\left(\rho_{\mathrm{c}} h+\frac{\rho_{\mathrm{f}} R}{2}\right) \frac{\partial^{2} w}{\partial t^{2}}+\frac{N_{\theta}}{R}+\rho_{\mathrm{f}} \frac{R}{2} U^{2} \frac{\partial^{2} w}{\partial x^{2}}+\rho_{\mathrm{f}} R U \frac{\partial^{2} w}{\partial x \partial t}=0$

where $t$ stands for the variable of time, $\rho_{f}$ and $\rho_{\mathrm{c}}$ are the mass density for fluid and CNT, respectively. Considering the geometric relationships under axisymmetric conditions $(\partial / \partial \theta=0)$, we have the following: $\left(\rho_{\mathrm{c}} h+\frac{\rho_{\mathrm{f}} R}{2}\right) \frac{\partial^{2} w}{\partial t^{2}}+\frac{N_{\theta}}{R}+\rho_{\mathrm{f}} \frac{R}{2} U^{2} \frac{\partial^{2} w}{\partial x^{2}}+\rho_{\mathrm{f}} R U \frac{\partial^{2} w}{\partial x \partial t}=0$.

Equations (12-14) are axisymmetric governing equations of motion for fluid-conveying CNTs based on membrane cylindrical shell theory. Here, the nanoscale effects contributed by CNTs are measured by stress nonlocallity and strain gradient, as shown in Eqs. (5-7). However, the scale effects leaded by fluid flow inside the CNTs are not negligible. Since slip boundary effect is inappropriate for liquids, the nonlocal fluid dynamic theory developed by Eringen is an acceptable option. According to nonlocal fluid dynamics principles, flow velocity, $U$, inside a nanotube is calculated as follows [17]:

$\eta \frac{\mathrm{d} U}{\mathrm{~d} r}=\frac{P R}{\operatorname{Si}(\lambda)}\left\{-\frac{1}{\lambda} \sin \lambda \sin \lambda \bar{r}+\frac{1}{2} \bar{y}[\operatorname{Si} \lambda(1+\bar{r})+\operatorname{Si} \lambda(1-\bar{r})]\right\}$,

where

$\operatorname{Si}(x)=\int_{0}^{x} \frac{\sin t}{t} \mathrm{~d} t, \lambda=\frac{R}{(\text { ea })_{f}}, \bar{r}=\frac{r}{R}, 0 \leq r \leq R$.

In Eqs. $(15,16), \eta$ is the fluid viscosity coefficient, $P$ is the pressure gradient, and (ea) $)_{f}$ stands for the nonlocal parameter for the fluid. Considering Eqs. (5-7), Eqs. (12-14) change as

$$
\begin{aligned}
\rho_{\mathrm{c}} h & {\left[\frac{\partial^{2} u}{\partial t^{2}}-(\mathrm{ea})^{2} \frac{\partial^{4} u}{\partial t^{2} \partial x^{2}}\right] } \\
& =\frac{\mathrm{Eh}}{1-v^{2}}\left[\left(\frac{\partial^{2} u}{\partial x^{2}}-l^{2} \frac{\partial^{4} u}{\partial x^{4}}\right)+\frac{v}{R}\left(\frac{\partial w}{\partial x}-l^{2} \frac{\partial^{3} w}{\partial x^{3}}\right)\right],
\end{aligned}
$$

$\rho_{\mathrm{c}} h \frac{\partial^{2} v}{\partial t^{2}}-G h \frac{\partial v}{\partial x}=0$

$\left(\rho_{\mathrm{c}} R h+\frac{\rho_{\mathrm{f}} R^{2}}{2}\right)\left[(\mathrm{ea})^{2} \frac{\partial^{4} w}{\partial x^{2} t^{2}}-\frac{\partial^{2} w}{\partial t^{2}}\right]+\frac{1}{2} \rho_{\mathrm{f}} R^{2} U^{2}\left[(\mathrm{ea})^{2} \frac{\partial^{4} w}{\partial x^{4}}-\frac{\partial^{2} w}{\partial x^{2}}\right]$

$+\rho_{\mathrm{f}} R U\left[(\mathrm{ea})^{2} \frac{\partial^{4} w}{\partial x^{3} \partial t}-\frac{\partial^{2} w}{\partial x \partial t}\right]=\frac{\mathrm{Eh}}{1-v^{2}}\left[\frac{1}{R}\left(w-l^{2} \frac{\partial^{2} w}{\partial x^{2}}\right)+v\left(\frac{\partial u}{\partial x}-l^{2} \frac{\partial^{3} u}{\partial x^{3}}\right)\right]$. $\varepsilon_{x}=\partial u / \partial x, \varepsilon_{\theta}=w / R, \gamma=\partial v / \partial x$,

where $u, v$ and $w$ are displacements in the $x, \theta$, and $r$ directions, respectively. Using Eq. (11), Eqs. (8-10) simplify to

$$
\begin{aligned}
& \rho_{\mathrm{c}} h \frac{\partial^{2} u}{\partial t^{2}}-\frac{\partial N_{x}}{\partial x}=0, \\
& \rho_{\mathrm{c}} h \frac{\partial^{2} v}{\partial t^{2}}-\frac{\partial N_{x \theta}}{\partial x}=0,
\end{aligned}
$$

Equations (15-19) are the governing equations of axisymmetric free vibration of fluid-conveying nanotubes on the basis of nonlocal fluid dynamic and nonlocal strain gradient. Obviously, Eq. (18) is not coupled with Eqs. $(17,19)$ in such a way because the torsional wave here is independent with the waves at longitudinal and radial direction. Now, if we only consider the axisymmetric wave motion governed by the coupled dynamic equations in terms of $u$ and $w$, we yield the following expression [35]:

$u=\bar{u} e^{\mathrm{i}(k x-\omega t)}$ 
$w=\bar{w} e^{\mathrm{i}(k x-\omega t)}$,

where $i=\sqrt{-1}$. Substituting Eqs. $(20,21)$ into Eqs. (17, 19), we obtain

$\left\{\frac{\mathrm{Eh}}{1-v^{2}}\left(k^{2}+l^{2} k^{4}\right)-\rho_{\mathrm{c}} h\left[1+(\mathrm{ea})^{2} k^{2}\right] \omega^{2}\right\}$

$\bar{u}-\mathrm{i} \frac{\mathrm{Eh}}{1-v^{2}} \frac{v}{R}\left(k+l^{2} k^{2}\right) \bar{w}=0$,

$\left\{\left(\rho_{\mathrm{c}} R h+\frac{\rho_{\mathrm{f}} R^{2}}{2}\right)\left[(\mathrm{ea})^{2} k^{2}+1\right] \omega^{2}+\rho_{\mathrm{f}} R U\left[(\mathrm{ea})^{2} k^{3}-k\right] \omega\right.$

$\left.+\frac{1}{2} \rho_{\mathrm{f}} R^{2} U^{2}\left[(\mathrm{ea})^{2} k^{4}+k^{2}\right]\right\} \bar{w}-\mathrm{i} \frac{E h v}{1-v^{2}}\left(l^{2} k^{3}+k\right) \bar{u}=0$.

Equations $(22,23)$ can be expressed as following:

$\left(\begin{array}{ll}\Delta_{1} & \Delta_{2} \\ \Delta_{3} & \Delta_{4}\end{array}\right)\left(\begin{array}{l}\bar{u} \\ \bar{w}\end{array}\right)=0$

where

$$
\begin{aligned}
\Delta_{1}= & \frac{\mathrm{Eh}}{1-v^{2}}\left(k^{2}+l^{2} k^{4}\right)-\rho_{\mathrm{c}} h\left[1+(\mathrm{ea})^{2} k^{2}\right] \omega^{2}, \\
\Delta_{2}= & -\mathrm{i} \frac{\mathrm{Eh}}{1-v^{2}} \frac{v}{R}\left(k+l^{2} k^{2}\right), \\
\Delta_{3}= & -\mathrm{i} \frac{E h v}{1-v^{2}}\left(l^{2} k^{3}+k\right), \\
\Delta_{4}= & \left(\rho_{\mathrm{c}} R h+\frac{\rho_{\mathrm{f}} R^{2}}{2}\right)\left[(\mathrm{ea})^{2} k^{2}+1\right] \omega^{2}+\rho_{\mathrm{f}} R U\left[(\mathrm{ea})^{2} k^{3}-k\right] \\
& \omega+\frac{1}{2} \rho_{\mathrm{f}} R^{2} U^{2}\left[(\mathrm{ea})^{2} k^{4}+k^{2}\right] .
\end{aligned}
$$

The existence of a non-zero solution for Eq. (24) requires

$$
\left|\begin{array}{ll}
\Delta_{1} & \Delta_{2} \\
\Delta_{3} & \Delta_{4}
\end{array}\right|=0
$$

Employing the dimensionless form below:

$$
\begin{aligned}
\bar{U} & =\left(\frac{\rho_{f} R}{\mathrm{Eh}}\right)^{1 / 2} U ; \xi=\frac{l}{L_{0}} ; \xi_{s}=\frac{(\mathrm{ea})_{s}}{L_{0}} ; \xi_{f} \\
& =\frac{(\mathrm{ea})_{f}}{L_{0}} ; \Omega=\left(\frac{\rho_{f} R}{\mathrm{Eh}}\right)^{1 / 2} \omega R ; K=k R .
\end{aligned}
$$

Equation (24) reduces into dimensionless form as follows:

$$
\left|\begin{array}{ll}
\bar{\Delta}_{1} & \bar{\Delta}_{2} \\
\bar{\Delta}_{3} & \bar{\Delta}_{4}
\end{array}\right|=0
$$

where

$$
\begin{aligned}
& \bar{\Delta}_{1}=\frac{\mathrm{Eh}}{\left(1-v^{2}\right) R^{2}}\left(K^{2}+\xi^{2} K^{4}\right)-\frac{\rho_{c} \mathrm{Eh}^{2}}{\rho_{f} R^{3}}\left(1+\xi_{c}^{2} K^{2}\right) \Omega^{2}, \\
& \bar{\Delta}_{2}=-\mathrm{i} \frac{\mathrm{Eh}}{1-v^{2}} \frac{v}{R}\left(\frac{K}{R}+\xi^{2} K^{2}\right), \\
& \bar{\Delta}_{3}=-\mathrm{i} \frac{E h v}{\left(1-v^{2}\right) R}\left(\xi^{2} K^{3}+K\right), \\
& \bar{\Delta}_{4}=\left(\frac{\rho_{\mathrm{c}} h}{R}+\frac{\rho_{\mathrm{f}}}{2}\right) \frac{\mathrm{Eh}}{\rho_{\mathrm{f}} R}\left(\xi_{\mathrm{c}}^{2} K^{2}+1\right) \Omega^{2}+\frac{E h}{R^{2}} \bar{U}\left(\xi_{\mathrm{c}}^{2} K^{3}-K\right) \Omega \\
& +\frac{E h}{2 R} \bar{U}^{2}\left(\xi_{\mathrm{c}}^{2} K^{4}+K^{2}\right) .
\end{aligned}
$$

By solving Eq. (26), the influence of scale effects on dynamic behaviors such as axisymmetric wave propagation and damping can be investigated in detail.

\section{Results and Discussion}

The dynamic characteristics of fluid-conveying CNTs could be investigated based on Eqs. (23-28). Some physics constants and parameters are defined as $P=1 \times 10^{3} \mathrm{~Pa} \mathrm{~m}^{-1}$; $R=1 \times 10^{-8} \mathrm{~m} ; h=0.045 \times 10^{-9} \mathrm{~m} ; \mu=1 \times 10^{6} \mathrm{~Pa} \cdot \mathrm{s}$; $L_{0}=1 \times 10^{-9} \mathrm{~m} ; E=1.0 \times 10^{12} \mathrm{~Pa} ; \rho_{f}=1 \times 10^{3} \mathrm{~kg} \cdot \mathrm{m}^{-3}$; $\rho_{\mathrm{c}}=2.27 \times 10^{3} \mathrm{~kg} \cdot \mathrm{m}^{-3}[31,32]$.

Figure 2 indicates the relationship between the firstmode frequency, $\Omega$, and $\xi$ for different values of $\xi_{\mathrm{c}}$. Here, the value of $\Omega$ decreases when $\xi_{\mathrm{c}}$ increases from 0.01 to 0.2 but increases when $\xi$ increases from 0 to 0.2 . Similar

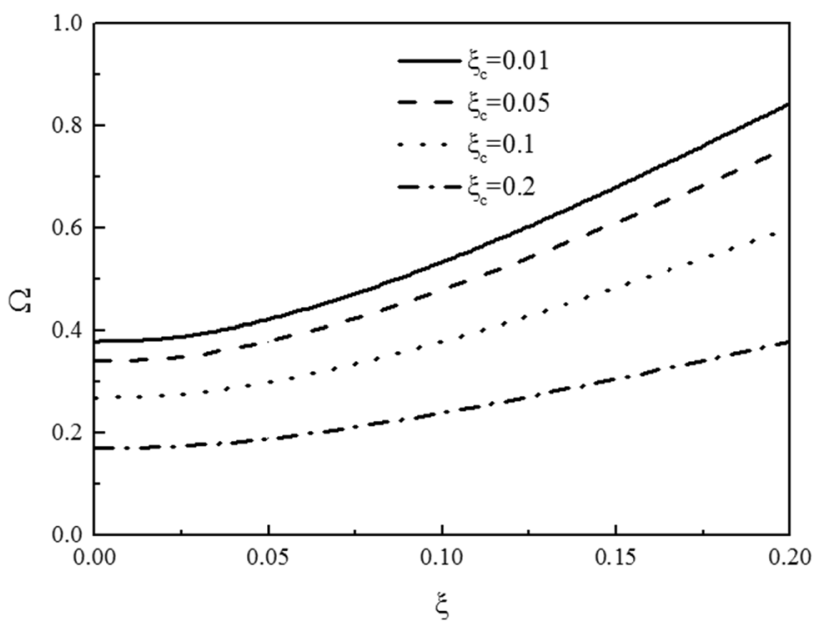

Fig. 2 First-mode $\Omega$ vs $\xi$ for different values of $\xi_{\mathrm{c}}$ 


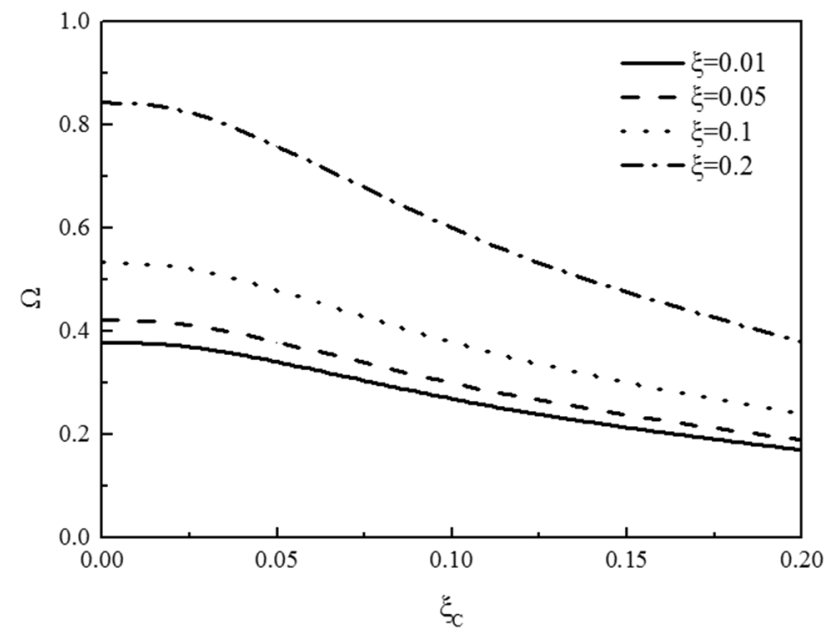

Fig. 3 First mode of $\Omega$ vs $\xi_{\mathrm{c}}$ for different values of $\xi$

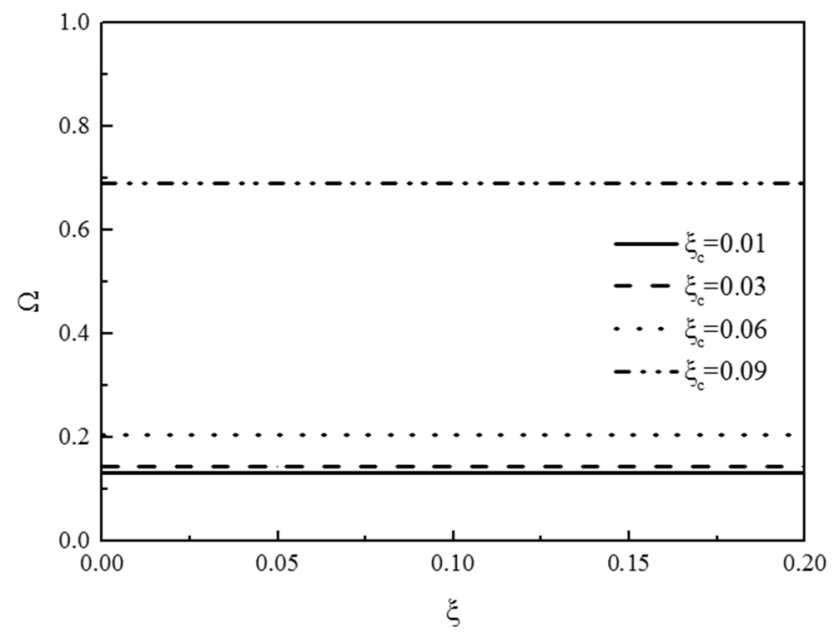

Fig. 4 Second-mode $\Omega$ vs $\xi$ for different values of $\xi_{\mathrm{c}}$

results are illustrated in Fig. 3, because the value of $\Omega$ continues to increase with decreasing $\xi_{\mathrm{c}}$ but increases when $\xi$ increases from 0.01 to 0.2 . Thus, wave propagation at first mode is promoted by strain gradient effects and damped by nonlocal stress effects.

However, different results for the second-mode wave are indicated in Figs. 4 and 5. First, the value of $\Omega$ keeps increasing when $\xi_{\mathrm{c}}$ rises from 0.01 to 0.09 in Fig. 4, but remains constant when $\xi$ decreases from 0 to 0.2 . Similar results are shown in Fig. 5, where $\Omega$ increases when $\xi_{\mathrm{c}}$ changes from 0 to 0.09 , but stays constant when $\xi$ takes different values. Therefore, the strain gradient effects do not influence second-mode wave propagation, but nonlocal stress effects promote wave propagation.

Figures 6 and 7 show the dispersion relation for firstmode wave propagation that $\Omega$ is a function of wavenumber $\mathrm{K}$ with different values of $\xi_{\mathrm{c}}$ and $\xi$, respectively. The

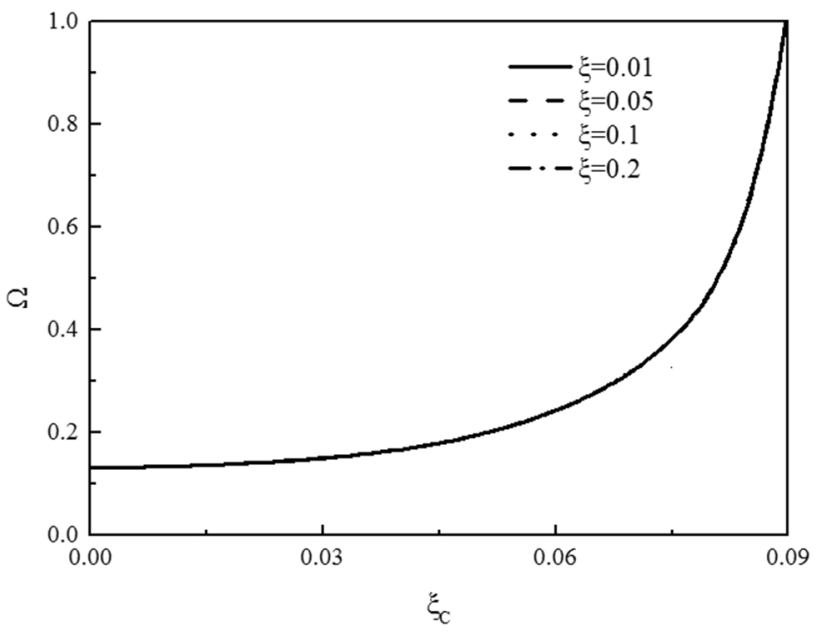

Fig. 5 Second-mode $\Omega$ vs $\xi_{\mathrm{c}}$ for different values of $\xi$

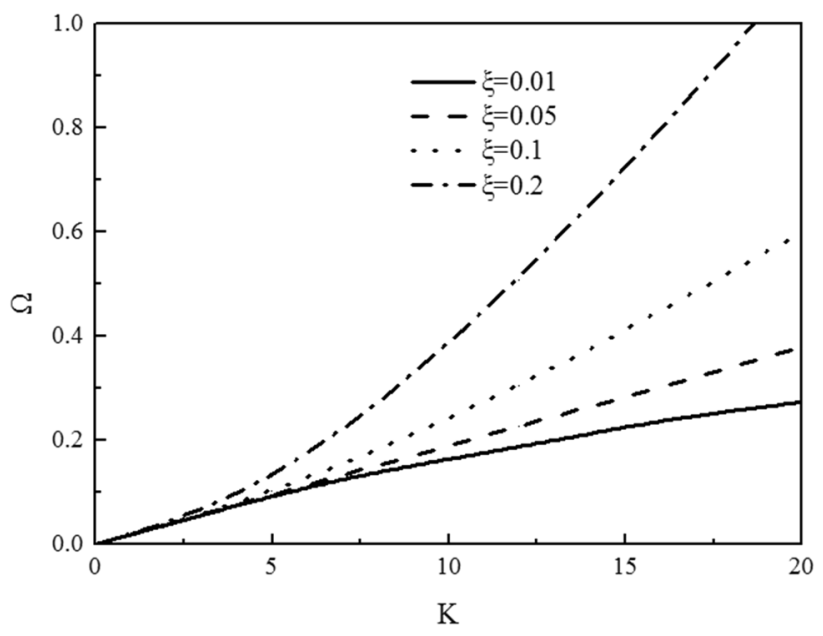

Fig. 6 First-mode $\Omega$ vs $K$ for different values of $\xi$

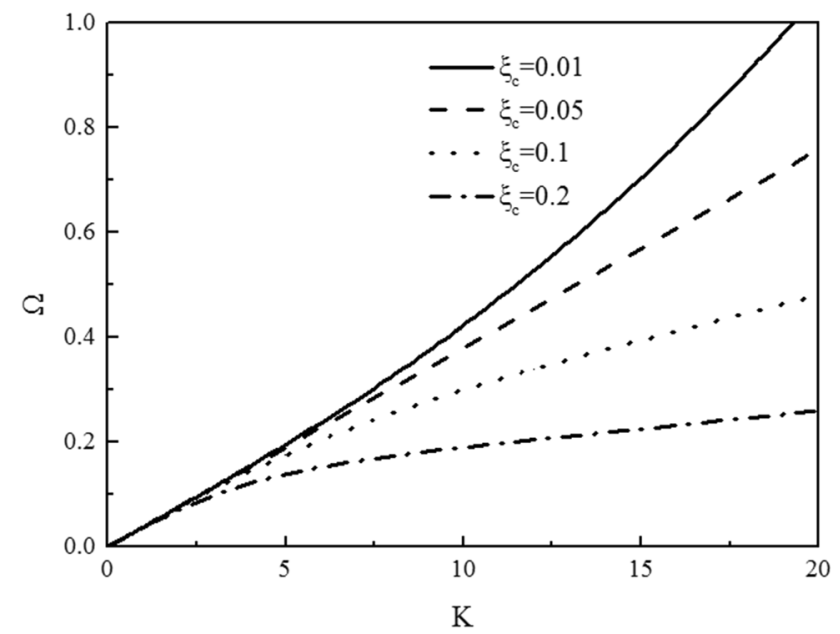

Fig. 7 First-mode $\Omega$ vs $K$ for different values of $\xi_{\mathrm{c}}$ 


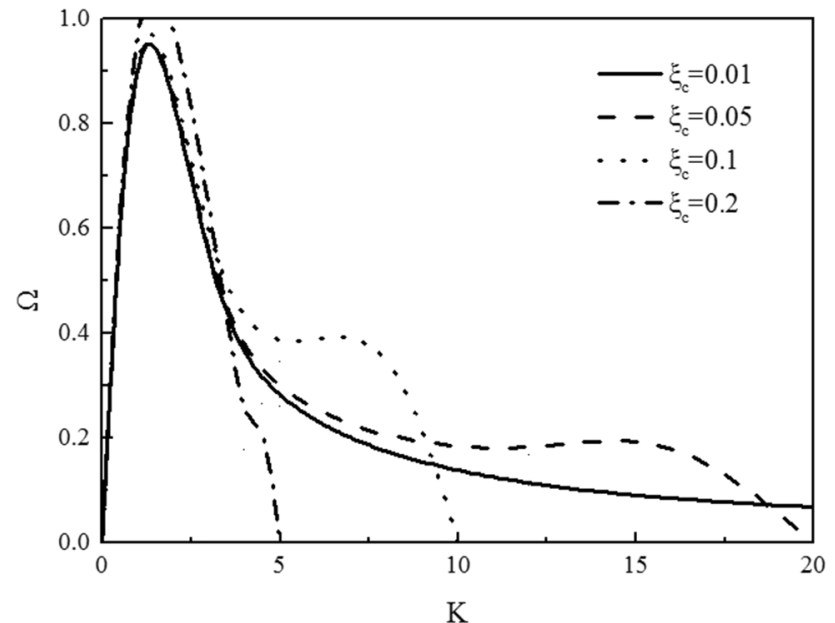

Fig. 8 Second-mode $\Omega$ vs $K$ with different values of $\xi_{\mathrm{c}}$

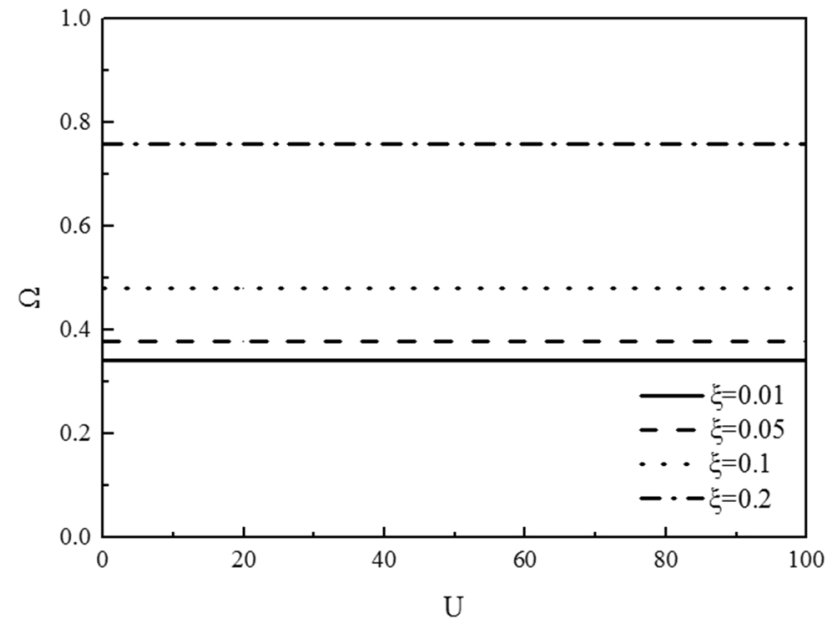

Fig. 9 First-mode $\Omega$ vs $U$ for different values of $\xi$

frequency grows with increasing wavenumber in Figs. 6 and 7 , which agrees with classical theory. Similar to our previous results, the effects of strain gradient and stress nonlocality have contrary influences on the first-mode wave propagation, because $\Omega$ keeps growing with increasing $\xi$ in Fig. 6, and declines with increasing $\xi_{\mathrm{c}}$ as seen in Fig. 7.

The dispersion relation for the second-mode wave is shown in Fig. 8 with different $\xi_{\mathrm{c}}$. The wave frequency increases with wavenumber $\mathrm{K}$ initially, then decreases when $\mathrm{K}$ goes beyond a critical value. Thus, waves with large wavelength and low wavenumber are promoted, and waves with low wavelength and high wave number are damped. The critical value of $K$ varies with $\xi_{\mathrm{c}}$ but remains between 2 and 3 , as shown in Fig. 8. Additionally, Fig. 8 shows that waves decay more rapidly when $\xi_{\mathrm{c}}$ increases and nonlocal effects are more powerful, which cause a stronger wave-decay effect.

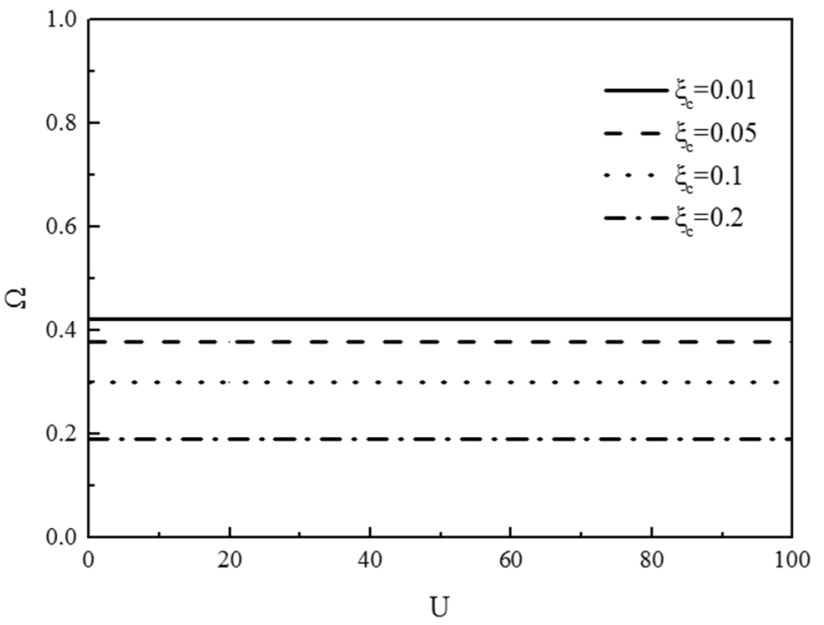

Fig. 10 First-mode $\Omega$ vs $U$ for different values of $\xi_{\mathrm{c}}$

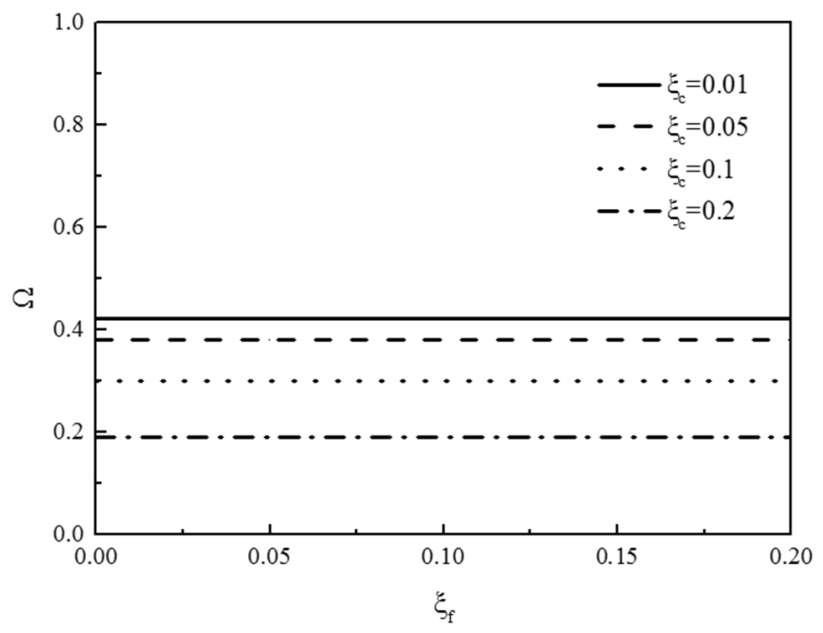

Fig. 11 First-mode $\Omega$ vs $\xi_{\mathrm{f}}$ for different values of $\xi_{\mathrm{c}}$

In addition to the effects dominated by strain gradient and stress nonlocality in CNTs, the results of the analysis for the nanoeffects contributed by inner fluid are also unnegligible. The influence of flow velocity $U$ on the first-mode wave is illustrated in Figs. 9 and 10. Both figures indicate that the flow velocity, $\mathrm{U}$, has no effect on the frequency, since $\Omega$ remains constant when $\mathrm{U}$ changes from 0 to 100 . Since $U$ is function of $\xi_{\mathrm{f}}$ according to Eq. (15), the nonlocal effects induced by fluid flow only affect the flow velocity. Thus, the first-mode $\Omega$ also stays constant for any value of $\xi_{\mathrm{f}}$, as shown in Fig. 11.

However, the second-mode of $\Omega$ is a function of $U$, as shown in Fig. 12. The value of $\Omega$ continues to increase when $U<10$, but decreases after $U>10$. This outcome can be explained as the fluid flow with low velocity promotes the wave propagation, while flow with high velocity leads to wave damping. 


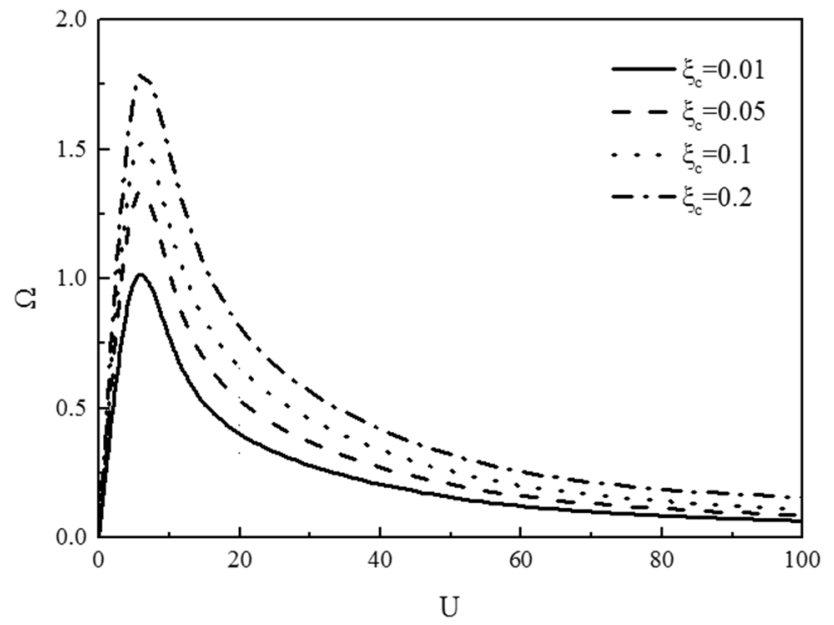

Fig. 12 Second-mode $\Omega$ vs $U$ for different values of $\xi_{\mathrm{c}}$

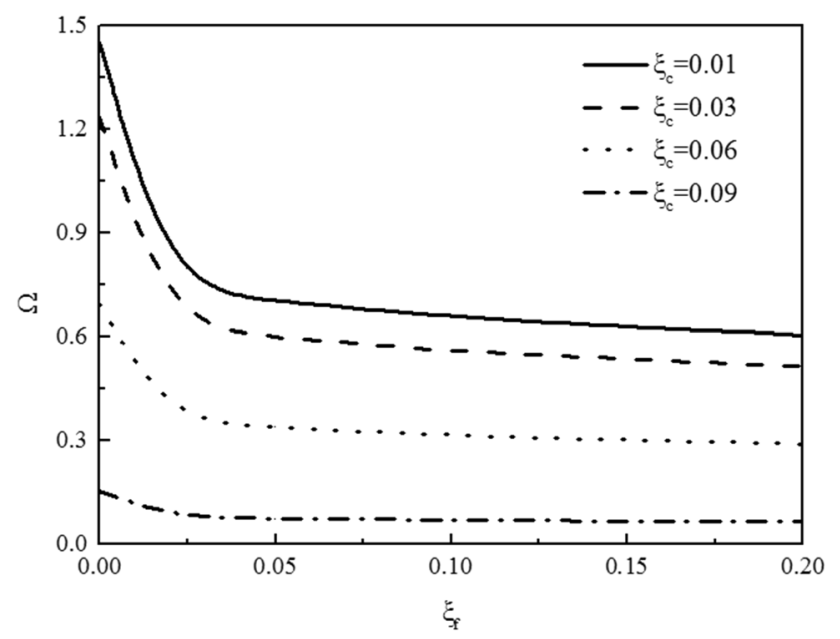

Fig. 13 Second-mode $\Omega$ vs $\xi_{\mathrm{f}}$ for different values of $\xi_{\mathrm{c}}$

Furthermore, the value of second-mode $\Omega$ only continues to decrease when $\xi_{f}$ increases, as shown in Fig. 13. Moreover, $\Omega$ decreases sharply when $\xi_{f}<0.03$ initially, then decreases more slowly and approaches a constant value. In contrast to the effects of stress nonlocality on CNTs, the fluid nonlocality leads to wave damping. As discussed previously, the nonlocal effects of fluid flow only affect flow velocity. Thus, the flow velocity decreases with increasing $\xi_{\mathrm{f}}$, and lower velocities cause weaker vibrations and wave frequencies.

\section{Conclusion}

A shell model on the basis of nonlocal strain gradient theory is developed to simulate axisymmetric wave propagation in fluid-conveying CNTs when the nanofluid effect is measured by nonlocal fluid dynamics. By solving the governing equations of axisymmetric wave, the wave characteristics are analyzed in detail. The numerical results and wave behaviors are indicated as follows:

(1) The nonlocal stress effect induces the damping of firstmode waves but promotes second-mode wave propagation.

(2) The strain gradient effect promotes the propagation of the first-mode wave but has no influence on second-mode waves.

(3) The nonlocal fluid effect leads to the damping of second-mode waves but exerts no influence on first-mode waves.

Therefore, three kinds of scale effect exert different influences on axisymmetric wave characteristic of fluid-conveying CNTs. These numerical conclusions are meaningful for the design and processing of MEMS.

Acknowledgements This work was supported by the National Natural Science Foundation of China (Grant No. 11962009).

Open Access This article is licensed under a Creative Commons Attribution 4.0 International License, which permits use, sharing, adaptation, distribution and reproduction in any medium or format, as long as you give appropriate credit to the original author(s) and the source, provide a link to the Creative Commons licence, and indicate if changes were made. The images or other third party material in this article are included in the article's Creative Commons licence, unless indicated otherwise in a credit line to the material. If material is not included in the article's Creative Commons licence and your intended use is not permitted by statutory regulation or exceeds the permitted use, you will need to obtain permission directly from the copyright holder. To view a copy of this licence, visit http://creativecommons.org/licenses/by/4.0/.

\section{References}

1. Karniadakis GE, Beskok A, Aluru N (2005) Microflows and nanoflows: fundamentals and simulation. Springer, New York

2. Lin JZ (2010) Micro-nano flow theory and its applications. Science Press, Beijing

3. Ji GH, Ji HM (2009) Microfluidic theory and elements. Higher Education Press, Beijing

4. Krishnan TVS, Babu JS, Sathian SP (2013) A molecular dynamics study on the effect of thermostat selection on the physical behavior of water molecules inside single walled carbon nanotubes. J Mol Liq 188:42-48

5. Wang JF, Xie HQ (2015) Molecular dynamic investigation on the structures and thermal properties of carbon nanotube interfaces. Appl Therm Eng 88:347-352

6. Liang F, Bao RD (2015) Fluid-structure interaction of microtubes conveying fluid considering thermal effect. J Vib Shock 34(5):141-144

7. Ghazavi MR, Molki H, Beigloo A (2018) Nonlinear analysis of the micro/nanotube conveying fluid based on second strain gradient theory. Appl Math Model 60:77-93

8. Yang Y, Zhang LX, Lim CW (2011) Wave propagation in double-walled carbon nanotubes on a novel analytically nonlocal Timoshenko-beam model. J Sound Vib 330(8):1704-1717 
9. Soleimani I, Beni YT (2018) Vibration analysis of nanotubes based on two-node size dependent axisymmetric shell element. Arch Civ Mech Eng 18(4):1345-1358

10. Yang Y, Zhang LX, Lim CW (2012) Wave propagation in fluidfilled single-walled carbon nanotube on analytically nonlocal Euler-Bernoulli beam model. J Sound Vib 331(7):1567-1579

11. Li C, Yu YM, Fan XL et al (2015) Dynamical characteristics of axially accelerating weak visco-elastic nanoscale beams based on a modified nonlocal continuum theory. J Vib Eng Technol 3(5):565-574

12. Li C (2016) On vibration responses of axially travelling carbon nanotubes considering nonlocal weakening effect. J Vib Eng Technol 4(2): 175-181

13. Li C, Zhang N, Fan XL et al (2019) Impact behaviors of cantilevered nano-beams based on the nonlocal theory. J Vib Eng Technol 7(5):533-542

14. Rashidi V, Mirdamadi HR, Shirani E (2012) A novel model for vibrations of nanotubes conveying nanoflow. Comp Maer Sci 51(1):347-352

15. Mirramezani M, Mirdamadi HR (2012) The effects of Knudsendependent flow velocity on vibrations of a nano-pipe conveying fluid. Arch Appl Mech 82(7):879-890

16. Mirramezani M, Mirdamadi HR (2012) Effects of nonlocal elasticity and Knudsen number on fluid-structure interaction in carbon nanotube conveying fluid. Physical E 44(10):2005-2015

17. Eringen AC (2002) Nonlocal continuum field theories. Springer, New York

18. Lim CW, Zhang G, Reddy JN (2015) A higher-order nonlocal elasticity and strain gradient theory and its applications in wave propagation. J Mech Phys Solids 78:298-313

19. Shen JP, Wang PY, Li C et al (2019) New observations on transverse dynamics of microtubules based on nonlocal strain gradient theory. Compos Struct 225:111036

20. Li C, Lai SK, Yang X (2019) On the nano-structural dependence of nonlocal dynamics and its relationship to the upper limit of nonlocal scale parameter. Appl Math Model 69:127-141

21. Liu JJ, Li C, Fan XL et al (2017) Transverse free vibration and stability of axially moving nanoplates based on nonlocal elasticity theory. Appl Math Model 45:65-84

22. Li L, Hu YJ, Li X et al (2016) Size-dependent effects on critical flow velocity of fluid-conveying microtubes via nonlocal strain gradient theory. Microfluid Nanofluid 20(5):76

23. Amiri A, Talebitooti R, Li L (2018) Wave propagation in viscousfluid-conveying piezoelectric nanotubes considering surface stress effects and Knudsen number based on nonlocal strain gradient theory. Eur Phys J Plus 133(7):252
24. Li L, Hu YJ, Wang XL et al (2018) Thermoelastic damping of graphene nanobeams by considering the size effects of nanostructure and heat conduction. J Therm Stresses 41(9):1182-1200

25. Zeighampour H, Yaghoub TB, Mohsen BD (2018) Wave propagation in viscoelastic thin cylindrical nanoshell resting on a viscoPasternak foundation based on nonlocal strain gradient theory. Thin Wall Struct 122:378-386

26. Zeighampour H, Yaghoub TB, Iman K (2017) Wave propagation in double-walled carbon nanotube conveying fluid considering slip boundary condition and shell model based on nonlocal strain gradient theory. Microfluid Nanofluid 21(5):85

27. Mahinzare M, Zeighampour H, Ghadiri M et al (2017) Sizedependent effects on critical flow velocity of a SWCNT conveying viscous fluid based on nonlocal strain gradient cylindrical shell model. Microfluid Nanofluid 21(7):123

28. Zeighampour H, Yaghoub TB (2014) Size-dependent vibration of fluid-conveying double-walled carbon nanotubes using couple stress shell theory. Phys E 61:28-39

29. Mohammadi K, Rajabpour A, Ghadiri M (2018) Calibration of nonlocal strain gradient shell model for vibration analysis of a CNT conveying viscous fluid using molecular dynamics simulation. Compos Mater Sci 148:104-115

30. Bahaadini R, Hosseini M, Jamali B (2018) Flutter and divergence instability of supported piezoelectric nanotubes conveying fluid. Phys B 529:57-65

31. Yang Y, Wang JR, Yan WH (2019) Study on dynamic characteristics of microchannel fluid-solid coupling systems in nonlocal stress fields. J Vib Eng Technol 7(5):477-485

32. Yang Y, Yan WH, Wang JR (2019) Study on the small-scale effect on wave propagation characteristics of fluid-filled carbon nanotubes based on nonlocal fluid theory. Adv Mech Eng 11(1):1-9

33. Wang Q (2006) Axi-symmetric wave propagation of carbon nanotubes with non-local elastic shell model. Int J Struct Stab Dyn 6(2):285-296

34. Paidoussis MP (1998) Fluid-structure interactions: slender structures and axial flow. Academic Press, San Diego

35. Peter H, Anirvan DG (2007) Vibrations and waves in continuous mechanical systems. John Wiley \& Sons Ltd, Chichester

Publisher's Note Springer Nature remains neutral with regard to jurisdictional claims in published maps and institutional affiliations. 